\title{
Effects of L-citrulline malate oral supplementation on post-exercise hypotension
}

\author{
Juliano Casonatto1, Renata Cristina Góes', Kamila Grandolfi', João Vagner Cavalari'
}

\begin{abstract}
Background: L-citrulline malate supplementation promotes an increase in oxide nitric metabolites, which are considered important mediators of peripheral vasodilation. Thus, the supplementation with this substance might maximize the duration and magnitude of post-exercise hypotension. Objectives: To investigate the hypotensive effect of L-citrulline malate supplementation following a single session of aerobic exercise in normotensives. Methods: Forty adults, normotensives, sedentary, were randomly assigned to one of the four experimental groups (control-placebo, control-Lcitrulline, exercise-placebo and exercise-Lcitrulline). All participants ingested a sachet with placebo or L-citrulline with malate (6 grams), both dissolved in water and the ingestion was made 120 minutes before starting the experimental session. The subjects performed 40 minutes of walk/run at $60-70 \%$ of reserve heart rate. For the control session, they remained seated in rest during 40 minutes. The blood pressure was taken in rest and every 10 minutes until complete 60 minutes after the experimental session. The ambulatory blood pressure device take the readings every 20 minutes (awake time) and each 30 minutes (sleep time) over the course of 24 hours. Results: L-citrulline malate supplementation associated to aerobic exercise promoted significantly reductions on systolic blood pressure when compared with control-placebo at 20 min (net change[NC]: $9.7 \mathrm{mmHg}[P=0.010]), 40 \mathrm{~min}$ (NC: $12.7 \mathrm{mmHg}[P=0.010]), 50 \mathrm{~min}$ (NC: $12.1 \mathrm{mmHg}[P=0.012]), 60 \mathrm{~min}$ (NC: $9.0 \mathrm{mmHg}$ $[P=0.050]$ ) and overall mean (NC: $7.8 \mathrm{mmHg}[P=0.018]$ ) post exercise. Conclusion: Acute L-citrulline malate supplementation might potentiate the post-exercise hypotension effects in normotensive subjects.
\end{abstract}

KEYWORDS: Blood Pressure, Dietary Supplements, Ambulatory Blood Pressure Monitoring.

\section{INTRODUCTION}

L-citrulline is a non-essential amino acid that has a key role in the arginine-nitric oxide system, increasing nitric oxide (NO) biodisponibility ${ }^{(1)}$. Low concentrations of L-citrulline can be provided by nutritional sources in regular food, but the main L-citrulline availability is endogenously produced by means of two differents ways: 1 ) NO coproduct (secondary amount) and 2) ornithine carbamylation (principal amount) by metabolites (glutamine, proline and arginine) in only two cells types (enterocytes and hepatocytes) ${ }^{(2)}$. The liver citrulline produce is all channeled to the urea cycle, thus, small or nothing amount of citrulline is directed to the circulation ${ }^{(3)}$. On the other hand, the enterocytes L-citrulline produced enter on circulation system, skip the liver, and goes to the kidney (and others tissues) to arginine sinthesys ${ }^{(4,5)}$. For this reason, is suggested that L-citrulline supplementation can be an efficient strategy to increase the extracellular L-arginine levels, which is recognized as the NO synthesis precursor ${ }^{(6)}$. In this line, some studies has indicated that L-citrulline supplementation increase plasmatic NO metabolites concentration $^{(7,8)}$, an important peripheral dilate mediator. Thus, is possible that L-citrulline supplementation improve the post-exercise hypotension effects (duration and/or magnitude).

To our knowledge, there is no other studies investigating the acute L-citrulline supplementation effects on the blood pressure, after aerobic exercises. Therefore, the assessment of this hypothesis can help to improve the non-pharmacological intervention strategies focusing the prevention and treatment of hypertension. Accordingly, the aim of the present study was to investigate whether L-citrulline supplementation might influence the acute post-exercise blood pressure response in normotensive subjects. We hypothesized that the acute post-exercise reduction in blood pressure will be more pronounced after L-citrulline supplementation. 


\section{METHODS}

\section{Participants}

After sample size calculation (see statistical analysis session), 40 normotensives sedentary subjects participated in the study. The volunteers, both gender, adults, without osteoarticular disabilities and they had a medical authorization to practice physical activity. Participants were recruited from an exercise program project linked to the university that offered stretching and functional exercises sessions to the external community. The study followed the Declaration of Helsinki and approved by the local Institution Ethics Committee (56500315.6.0000.0108). All participants were informed about the methods before giving written informed consent. Table 1 shows the basics characteristics of the participants. The weight was measured by the digital anthropometric scale (Urano, OS $180 \mathrm{~A}$, Canoas, Brazil), with accuracy of $0.1 \mathrm{~kg}$ and height was measured by stadiometer with accuracy of $0.1 \mathrm{~cm}$, accordance to the procedures described by Gordon et a $\left.\right|^{(9)}$. The body mass index (BMI) was defined as body mass ( $\mathrm{kg}$ ) divided by the square of the body height.

\section{Laboratory Blood Pressure (laboratorial phase)}

Laboratory blood pressure was measured by using an oscillometric monitor (Omron MX3 Plus, Bannockburn, EUA) previously validated for clinic measures in adults ${ }^{(10)}$. Firstly, the participants remained seated (rest period) in calm, quiet and thermoneutral $\left(22^{\circ}-24^{\circ} \mathrm{C}\right)$ environment for $20 \mathrm{~min}$. The blood pressure was measured three times during the rest period (at $10 \mathrm{~min}, 15 \mathrm{~min}$ and $20 \mathrm{~min}$ ). The rest blood pressure value was considered as the average of these three measurements. Immediately following the sessions (exercise or control), the blood pressure was measured in a quite environment during 60 minutes. The blood pressure measurements were taking according to the American Heart Association recommendations ${ }^{(11)}$.

\section{Ambulatory blood pressure monitoring (ambulatory phase)}

The ambulatory blood pressure was measured by an oscillometric device (Dyna-MAPA - São Paulo, Brazil) attached on the left arm, always by the same investigator, in accordance with procedures described by American Heart Association ${ }^{(11)}$. The participants received instructions to keep the arm stand during the measures. The device was calibrated by direct comparison with mercury sphygmomanometer, by trained technical person, in agreement with recommendations ${ }^{(11)}$. The monitor was setting to register the systolic and diastolic blood pressure and heart rate every 20 minutes, except during nighttime $(23 \mathrm{~h} 00 \mathrm{~min}$ to $08 \mathrm{~h} 00 \mathrm{~min}$ ) that the readings were taken every 30 minutes, to reduce sleep disturbs. The device screen was blinded to the subject to avoid feedback. All participants were instructed to register and reported their sleep time in a diary on the day after. The data was recorded in the device memory then they were send to a computer using specific software (Aplication Dyna Mapa - Version 5.0.382.12) for analysis. The average of the valid readings were above $90 \%$ for all participants. The Delta between rest period and mean of ABPM periods (awake, asleep and 24 hours) was calculated for analysis.

\section{Study design}

The study was an acute, randomized, double-blind, placebo-controlled to evaluate the effects of Lcitrulline malate supplementation on post-exercise hypotension. The participants were randomly allocated (using a random number table - https://www.random.org/) in four different experimental groups (Exercise-Lcitrulline; Exercise-Placebo; Control-Lcitrulline; Control-Placebo). The L-citrulline supplementation was done in double-blind format. The participants ingested a sachet which contained L-citrulline malate ( 6 grams) or placebo ( 6 grams of corn starch) dissolved in water. The substances were ingested 120 minutes before the experimental session or control. Anthropometric measures were taken before the rest period. The exercise

Table 1. Characteristics of participants.

\begin{tabular}{|c|c|c|c|c|c|c|c|c|}
\hline & \multicolumn{2}{|c|}{ Control-Placebo } & \multicolumn{2}{|c|}{ Control-Lcitrulline } & \multicolumn{2}{|c|}{ Exercise-Placebo } & \multicolumn{2}{|c|}{ Exercise-Lcitrulline } \\
\hline & Mean & SE & Mean & SE & Mean & SE & Mean & SE \\
\hline Age (years) & 30.4 & $(2.5)$ & 27.3 & $(1.6)$ & 27.7 & $(2.3)$ & 29.9 & $(2.4)$ \\
\hline Body mass (kg) & 68.2 & $(3.6)$ & 69.7 & $(3.0)$ & 70.8 & $(6.1)$ & 76.4 & $(4.5)$ \\
\hline Height (m) & 1.68 & $(0.02)$ & 1.67 & $(0.02)$ & 1.69 & $(0.03)$ & 1.71 & $(0.02)$ \\
\hline $\mathrm{BMI}$ & 23.9 & $(0.8)$ & 24.7 & $(0.8)$ & 24.4 & $(1.4)$ & 25.9 & $(1.0)$ \\
\hline $\mathrm{WC}(\mathrm{cm})$ & 78.7 & $(2.0)$ & 80.5 & $(3.0)$ & 79.2 & $(4.3)$ & 84.3 & $(3.5)$ \\
\hline SBP (rest) & 113.1 & $(2.6)$ & 116.6 & $(4.7)$ & 116.3 & $(5.4)$ & 119.8 & $(3.6)$ \\
\hline DBP (rest) & 71.9 & $(1.4)$ & 75.6 & (3.1) & 71.5 & (3.0) & 73.9 & $(2.5)$ \\
\hline
\end{tabular}

Note: $\mathrm{SE}=$ standard error; $\mathrm{BMI}=$ body mass index; $\mathrm{WC}=$ waist circumference; $\mathrm{SBP}=$ systolic blood pressure; $\mathrm{DBP}=$ diastolic blood pressure. 
session consisted by 5 min to warm up ( $50 \%$ of $65 \%$ HRreserve) and 40 minutes of run/walk at $60-70 \%$ HRreserve in a treadmill. This was followed by a progressive reduction of the speed until the subject stop walk lasting 5 minutes (cooldown). In the control session, the participants remained seated for $40 \mathrm{~min}$ in calm environment and read was allowed. After the exercise/control sessions, the blood pressure was measured each 10 minutes over the course of 60 minutes and the heart rate variability was recorded continuously for 60 minutes (laboratorial phase). Then, 15 minutes was given to the participants to take a shower and change the clothes before the ambulatory blood pressure device be attached on their arm. The ambulatory blood pressure was recorded during 24 hours. In the next day, the participants were asked to return to the laboratory to remove the device. Data collection occurred between January and July of 2017.

\section{Statistical analysis}

Assuming a standard deviation of $5 \mathrm{mmHg}{ }^{(12)}$ for the systolic blood pressure, an alpha of $5 \%$ and a desired statistical power of $80 \%$, detecting a minimum difference of $7 \mathrm{mmHg}$ ${ }^{(12)}$ required 7 subjects in each group. The data are reported as mean and standard error. The sphericity Mauchly's test was applied. Greenhouse-Geisser correction was used if necessary. Then, these data were compared with a one-factor repeated measures general linear model (GLM). Fisher multiple comparisons were employed to examine differences between pairs of trials. The ambulatory blood pressure was analyzed as awake, sleep and 24 hours of monitoring. Statistical significance was defined as $P<0.05$. The statistical analysis was generated using SPSS, version 20, system for windows (SPSS/IBM, Chicago, IL, USA).

\section{RESULTS}

Participants characteristics and rest blood pressure values are shown in Table 1. The four experimental groups were not different for age, weight, height, body mass index, waist circumference and systolic/diastolic rest blood pressure. As shown in Table 2, Exercise-Lcitrulline group showed a significant reduction in systolic blood pressure when compared with control-placebo at $20 \mathrm{~min}$ (net change: $9.7 \mathrm{mmHg}[P=0.010]$ ), $40 \mathrm{~min}$ (net change: $12.7 \mathrm{mmHg}$ [ $P=0.010]$ ), $50 \mathrm{~min}$ (net change: $12.1 \mathrm{mmHg}$ [P=0.012]), $60 \mathrm{~min}$ (net change: $9.0 \mathrm{mmHg}[P=0.050]$ ) and overall mean (net change: $7.8 \mathrm{mmHg}[P=0.018]$ ) post exercise. Exercise-Lcitrulline group showed others significant reductions in systolic blood pressure when compared with Control-Lcitrulline at $40 \mathrm{~min}$ (net change: $11.6 \mathrm{mmHg}$ [ $P=0.018]$ ) and $50 \mathrm{~min}$ (net change: $9.0 \mathrm{mmHg}$ [P=0.049]) post exercise. Exercise-Placebo group showed a significant reductions in systolic blood pressure when compared with Control-Placebo at $20 \mathrm{~min}$ (net change: $9.0 \mathrm{mmHg}[P=0.015]$ ), $40 \mathrm{~min}$ (net change: $10.6 \mathrm{mmHg}[P=0.030]$ ) and overall mean (net change: $6.8 \mathrm{mmHg}[P=0.037]$ ) post exercise. Exercise-Placebo group showed other significant reduction in systolic blood pressure when compared with Control-Lcitrulline at $40 \mathrm{~min}$ (net change: $9.5 \mathrm{mmHg}[P=0.050]$ ). Moment-by-moment changes are show in Table 2.

As we can see in Table 3, no differences were observed to diastolic blood pressure in either of the groups at any of the moments. Regarding the pressure values obtained by ABPM, no differences were observed to systolic and diastolic blood

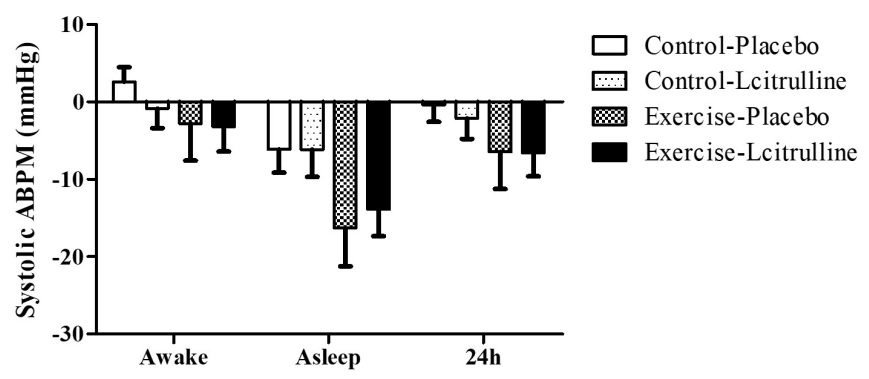

Figure 1: Systolic ambulatory blood pressure monitoring (ABPM) in awake, asleep and 24 hours periods.

Table 2. Systolic blood pressure post-exercise changes.

\begin{tabular}{|c|c|c|c|c|c|c|c|c|}
\hline & \multicolumn{2}{|c|}{ Control-Placebo } & \multicolumn{2}{|c|}{ Control-Lcitrulline } & \multicolumn{2}{|c|}{ Exercise-Placebo } & \multicolumn{2}{|c|}{ Exercise-Lcitrulline } \\
\hline & Mean & SE & Mean & SE & Mean & SE & Mean & SE \\
\hline $10 \mathrm{~min}$ & 3.4 & (1.9) & 0.5 & (3.4) & 0.3 & $(2.8)$ & 3.2 & (3.2) \\
\hline $20 \mathrm{~min}$ & 6.4 & (1.9) & 1.4 & (2.8) & -2.6 & $(3.1)^{*}$ & -3.3 & $(1.9)^{*}$ \\
\hline $30 \mathrm{~min}$ & -0.7 & $(4.0)$ & 0.2 & (3.0) & -3.7 & (3.6) & -4.6 & (2.3) \\
\hline $40 \mathrm{~min}$ & 6.0 & (3.6) & 4.9 & (3.1) & -4.6 & $(3.8)^{*+}$ & -6.7 & $(2.5)^{*+}$ \\
\hline $50 \mathrm{~min}$ & 5.1 & (3.7) & 2.0 & (2.7) & -2.3 & (3.2) & -7.0 & $(3.2)^{*+}$ \\
\hline $60 \mathrm{~min}$ & 2.2 & (2.9) & -2.1 & (4.1) & -5.9 & (3.4) & -6.8 & $(1.8)^{*}$ \\
\hline Mean $60 \mathrm{~min}$ post & 3.7 & (1.7) & 1.1 & (2.3) & -3.1 & $(3.0)^{*}$ & -4.1 & $(1.6)^{*}$ \\
\hline
\end{tabular}

Note: $\mathrm{SE}=$ standard error. ${ }^{*}=P<.05$ vs Control-Placebo; $\dagger=P<.05$ vs Control-Lcitrulline. 
Table 3. Diastolic blood pressure post-exercise changes.

\begin{tabular}{|c|c|c|c|c|c|c|c|c|}
\hline & \multicolumn{2}{|c|}{ Control-Placebo } & \multicolumn{2}{|c|}{ Control-Lcitrulline } & \multicolumn{2}{|c|}{ Exercise-Placebo } & \multicolumn{2}{|c|}{ Exercise-Lcitrulline } \\
\hline & Mean & SE & Mean & SE & Mean & SE & Mean & SE \\
\hline $10 \mathrm{~min}$ & 2.4 & 2.2 & 5.1 & 3.7 & 3.5 & 1.3 & 8.7 & 4.3 \\
\hline $20 \mathrm{~min}$ & 1.6 & 2.1 & 4.7 & 3.9 & 4.4 & 2.9 & 2.5 & 3.1 \\
\hline $30 \mathrm{~min}$ & -2.5 & 3.4 & -0.8 & 1.9 & 0.6 & 3.7 & 1.2 & 2.7 \\
\hline $40 \mathrm{~min}$ & 2.3 & 1.9 & 3.4 & 2.4 & 0.7 & 1.4 & 0.7 & 2.3 \\
\hline $50 \mathrm{~min}$ & 3.1 & 3.2 & 3.0 & 2.2 & 1.7 & 2.4 & 1.3 & 2.9 \\
\hline $60 \mathrm{~min}$ & 0.7 & 3.4 & 1.6 & 1.8 & -2.3 & 2.8 & 3.6 & 2.2 \\
\hline Mean 60 min post & 1.2 & 1.9 & 2.8 & 1.6 & 1.4 & 1.6 & 3.0 & 2.5 \\
\hline
\end{tabular}

Note: $\mathrm{SE}=$ standard error

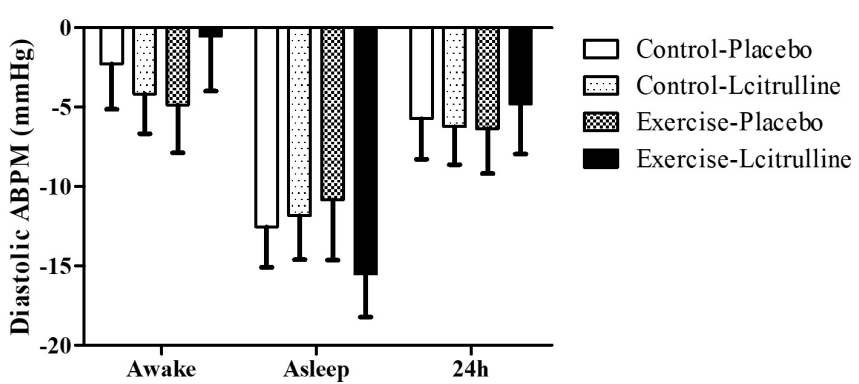

Figure 2: Diastolic ambulatory blood pressure monitoring (ABPM) in awake, asleep and 24 hours periods.

pressure in either of the groups for awake, asleep and 24 hours periods (Figure 1 and 2).

\section{DISCUSSION}

In the present study, we are showing that the post-exercise hypotension might be pronounced by L-citrulline malate supplementation in normotensives. The main finding of this randomized clinical trial was that the L-citrulline malate supplementation induce consistent post exercise blood pressure reduction during the first hour. This association (exercise + L-citrulline malate supplementation) showed consistent effects immediately after exercise (Table 2). Thus, based on our findings and previous studies in the literature, it is possible speculate that a sustained vasodilation in the L-citrulline-exercise group was caused by nitric oxide increase $^{(13)}$ and it was the main mechanism of blood pressure reduction. Unfortunately, in the present study we do not have evaluated these important biomarkers such as nitrite and nitrate. On the other hand, these incremental effects do not last long, like over the course of 24 hours. Studies involving L-citrulline supplementation are primarily connected to performance ${ }^{(14-19)}$. Thus, the investigations has shown that the L-citrulline is related with improving exercise tolerance ${ }^{(14,}$ $15,19)$. To our knowledge, the purpose of this study appears to be unpublished, thus, it is difficult compare this findings with others studies.
To date, only one randomized clinic trial was found ${ }^{(20)}$. The authors investigated the post-exercise blood pressure response 10 minutes after resistance exercise with L-citrulline malate supplementation ( 8 grams). However, they did not identified significant changes ${ }^{(20)}$. In the present study, we observed the hypotensive effects following 20 minutes post-exercise. Further, on the previous investigation ${ }^{(20)}$, the participants were submitted to resistance exercise session, which is an important methodological difference that needs to be addressed, as in the present study the participants were asked to perform an aerobic treadmill exercise session. It is well established that aerobic exercise is an efficient strategy to reduce blood pressure $(5-15 \mathrm{mmHg}$ ) comparing to resistance training $(5 \mathrm{mmHg})^{(21-24)}$, thus, the differences between modes of exercise do not allow for a direct comparison.

Regarding post exercise hypotension duration the literature is very divergent. According to some investigators ${ }^{(25,26)}$, the post exercise hypotension after aerobic exercise does not last for 24 hours, even in hypertensives who present greater post exercise hypotension magnitude ${ }^{(25,27)}$. Similarly, and as expected, no differences were identified in the awake, asleep and over the course of 24 hours periods. Thus, the Lcitrulline malate supplementation did not change the direction of what is already identified in the literature. Concerning to the mechanisms that might be involved in this response, we hypothesized that the vascular resistance reduction due to increased nitric oxide concentration could potentiate the post-exercise hypotension. However, we have not evaluated the mechanisms at this moment. Independently of the mechanisms that are involved in this response, the present study demonstrated that the exercise plus L-citrulline (intermediate of nitric oxide metabolism) causes a better consistence hypotensive effect. This finding suggests that the NO might be involve in this response and it might be over stimulated by exercise plus L-citrulline.

After these interesting findings with the combination of $\mathrm{L}$-citrulline and aerobic exercise, it is recommended that future studies include the measurements of nitric oxide availability, such as nitrite and nitrate. These measurements associated 
to the evaluation of important mechanisms such as peripheral vascular resistance and cardiac output might help us to understand the L-citrulline action. Further, considering this promising results, others studies can associated L-citrulline with others potential vasodilators nutrients and exercise modes enabling the development of adjuvant protocols to the hypertension treatment.

\section{CONCLUSION}

The results of this study suggest that acute L-citrulline malate supplementation is able to improve the post-exercise hypotensive effects in normotensives. Further studies involving chronic exercise and L-citrulline supplementation are needed and it might be a promising non-pharmacological therapeutic to avoid the hypertension.

\section{AUTHORS' CONTRIBUTIONS}

The study was designed by JC; data were collected and analyzed by JC, RCG, KG and JVC; data interpretation and manuscript preparation were undertaken by JC, RCG, KG and JVC. All authors approved the final version of the paper.

\section{CONFLICTS OF INTEREST}

The authors declares that there is no conflicts of interest regarding the publication of this article.

\section{REFERENCES}

1. Breuillard C, Cynober L, Moinard C. Citrulline and nitrogen homeostasis: an overview. Amino acids. 2015;47(4):685-91.

2. Marini JC, Didelija IC, Castillo L, Lee B. Glutamine: precursor or nitrogen donor for citrulline synthesis? American journal of physiology Endocrinology and metabolism. 2010;299(1):69-79.

3. Marini JC. Interrelationships between glutamine and citrulline metabolism. Current opinion in clinical nutrition and metabolic care. 2016;19(1):62-6.

4. Marini JC, Didelija IC, Fiorotto ML. Extrarenal citrulline disposal in mice with impaired renal function. American journal of physiology Renal physiology. 2014;307(6):F660-5.

5. Marini JC, Didelija IC. Arginine depletion by arginine deiminase does not affect whole protein metabolism or muscle fractional protein synthesis rate in mice. PloS one. 2015;10(3):e0119801.

6. Cleroux J, Kouame N, Nadeau A, Coulombe D, Lacourciere Y. After reffects of exercise on regional and systemic hemodynamics in hypertension. Hypertension. 1992;19(2):183-91.

7. Sureda A, Cordova A, Ferrer MD, Perez G, Tur JA, Pons A. L-citrulline-malate influence over branched chain amino acid utilization during exercise. Eur J Appl Physiol. 2010;110(2):341-51.

8. Sureda A, Cordova A, Ferrer MD, Tauler P, Perez G, Tur JA, et al. Effects of L-citrulline oral supplementation on polymorphonuclear neutrophils oxidative burst and nitric oxide production after exercise. Free Radic Res. 2009;43(9):828-35.

9. Gordon CC, Chumlea WC, Roche AF. Stature, recumbent length, and weight. Lohman TG, Roche AF, Martorell R, editors. Champaign: Human Kinetics Books; 1988;3-8.

10. Coleman A, Freeman P, Steel S, Shennan A. Validation of the Omron MX3 Plus oscillometric blood pressure monitoring device according to the European Society of Hypertension international protocol. Blood Press Monit. 2005;10(3):165-8.
11. Pickering TG, Hall JE, Appel LJ, Falkner BE, Graves J, Hill MN, et al. Recommendations for blood pressure measurement in humans and experimental animals: Part 1: blood pressure measurement in humans: a statement for professionals from the Subcommittee of Professional and Public Education of the American Heart Association Council on High Blood Pressure Research. Hypertension. 2005;45(1):142-61.

12. Casonatto J, Tinucci T, Dourado AC, Polito M. Cardiovascular and autonomic responses after exercise sessions with different intensities and durations. Clinics (Sao Paulo). 2011;66(3):453-8.

13. McKinley-Barnard S, Andre T, Morita M, Willoughby DS. Combined L-citrulline and glutathione supplementation increases the concentration of markers indicative of nitric oxide synthesis. J Int Soc Sports Nutr. 2015;12:27.

14. Glenn JM, Gray M, Jensen A, Stone MS, Vincenzo JL. Acute citrullinemalate supplementation improves maximal strength and anaerobic power in female, masters athletes tennis players. European journal of sport science. 2016;16(8):1095-103.

15. Suzuki T, Morita M, Kobayashi Y, Kamimura A. Oral L-citrulline supplementation enhances cycling time trial performance in healthy trained men: Double-blind randomized placebo-controlled 2-way crossover study. J Int Soc Sports Nutr. 2016;13:6.

16. Wong A, Alvarez-Alvarado S, Jaime SJ, Kinsey AW, Spicer MT, Madzima $\mathrm{TA}$, et al. Combined whole-body vibration training and I-citrulline supplementation improves pressure wave reflection in obese postmenopausal women. Applied physiology, nutrition, and metabolism Physiologie appliquee, nutrition et metabolisme. 2016;41(3):292-7.

17. Cunniffe B, Papageorgiou M, O'Brien B, Davies NA, Grimble GK, Cardinale M. Acute Citrulline-Malate Supplementation and High-Intensity Cycling Performance. J Strength Cond Res. 2016;30(9):2638-47.

18. Bailey SJ, Blackwell JR, Lord T, Vanhatalo A, Winyard PG, Jones AM. I-Citrulline supplementation improves $\mathrm{O} 2$ uptake kinetics and highintensity exercise performance in humans. J Appl Physiol (1985). 2015;119(4):385-95.

19. Cutrufello PT, Gadomski SJ, Zavorsky GS. The effect of I-citrulline and watermelon juice supplementation on anaerobic and aerobic exercise performance. Journal of sports sciences. 2015;33(14):1459-66.

20. Wax B, Kavazis AN, Luckett W. Effects of Supplemental Citrulline-Malate Ingestion on Blood Lactate, Cardiovascular Dynamics, and Resistance Exercise Performance in Trained Males. Journal of dietary supplements. 2016;13(3):269-82.

21. Kelley GA, Kelley KS. Progressive resistance exercise and resting blood pressure: A meta-analysis of randomized controlled trials. Hypertension. 2000;35(3):838-43.

22. Cornelissen VA, Smart NA. Exercise training for blood pressure: a systematic review and meta-analysis. J Am Heart Assoc. 2013;2(1):e004473.

23. Anunciação PG, Casonatto J, Polito M. Blood pressure responses and heart rate variability after resistance exercise with different intensities and same workload. Int Sport J. 2011;12(2):53-67.

24. Casonatto J, Goessler KF, Cornelissen VA, Cardoso JR, Polito MD. The blood pressure-lowering effect of a single bout of resistance exercise: A systematic review and meta-analysis of randomised controlled trials. European journal of preventive cardiology. 2016;23(16):1700-14.

25. Forjaz CL, Tinucci T, Ortega KC, Santaella DF, Mion D, Jr., Negrao CE. Factors affecting post-exercise hypotension in normotensive and hypertensive humans. Blood Press Monit. 2000;5(5-6):255-62.

26. Wallace JP, Bogle PG, King BA, Krasnoff JB, Jastremski CA. A comparison of 24-h average blood pressures and blood pressure load following exercise. Am J Hypertens. 1997;10(7 Pt 1):728-34.

27. Bermudes AM, Vassallo DV, Vasquez EC, Lima EG. Ambulatory blood pressure monitoring in normotensive individuals undergoing two single exercise sessions: resistive exercise training and aerobic exercise training. Arq Bras Cardiol. 2004;82(1):65-71. 